\title{
MORFOLOGÍA DE LOS GENITALES MASCULINOS DE TRES ESPECIES DE MUSCIDAE (INSECTA: DIPTERA) DE IMPORTANCIA FORENSE EN COLOMBIA*
}

\author{
Karen Viviana Solano ${ }^{1}$, Manuel Alejandro Ramírez-Mora ${ }^{2}$
}

\section{Resumen}

La entomología forense utiliza la biología y ecología de los insectos en casos médico-legales. Para su adecuada aplicación en este contexto, es necesario hacer una correcta identificación taxonómica de los especímenes relacionados con un caso en cualquier estado de desarrollo que se hayan encontrado. Frecuentemente en esta área se obtienen muestras incompletas o deterioradas de los adultos, dificultando su identificación. Una de las familias comúnmente encontrada en el contexto forense es Muscidae. El objetivo de este estudio es describir e ilustrar las características morfológicas de los genitales de machos adultos de Biopyrellia bipuncta (Wiedemann, 1830), Musca domestica Linnaeus, 1758 y Synthesiomyia nudiseta (Wulp, 1883), especies con importancia forense en Colombia. Se describen e ilustran caracteres genitales masculinos claves para la identificación morfológica: esternito V, la placa cercal, el surstilo y el aedeago. Adicionalmente, se proporcionan diagnosis complementarias de características morfológicas externas.

Palabras clave: entomología forense, identificación, M. domestica, B. bipuncta, S. nudiseta.

\section{MORPHOLOGY OF THE MALE GENITALIA OF THREE SPECIES OF MUSCIDAE (INSECTA: DIPTERA) OF FORENSIC IMPORTANCE IN COLOMBIA}

\begin{abstract}
Forensic entomology employs the biology and ecology of insects in medico-legal cases. For a precise application in this context, it is necessary to make a correct taxonomic identification in any developmental stage of the specimens related to a case. Incomplete or damaged samples of adults are often obtained from the case scene, making taxonomical identification unlikely. Muscidae is one of the families commonly found in the forensic context. The objective of this study is to describe and to illustrate the morphological characteristics of the adult male genitalia of Biopyrellia bipuncta (Wiedemann, 1830), Musca domestica Linnaeus, 1758 and Synthesiomyia nudiseta (Wulp, 1883), species with forensic importance in Colombia. Male genitalia structures like V sternite, the cercal plate, the surstyli and aedeagus are described and illustrated. Additionally complementary diagnosis of external morphological characteristics are provided.
\end{abstract}

Key words: Forensic entomology, identification, M. domestica, B. bipuncta, S. nudiseta.

\footnotetext{
${ }^{*}$ FR: 18-III-2014 . FA: 26-X-2014.

${ }_{1}$ Tecnóloga en Investigación Judicial, Grupo de Investigación en Ciencias Forenses y Salud, Tecnológico de Antioquia, Institución Universitaria. Medellín, Colombia. E-mail: karen_ljspt@yahoo.es

${ }^{2}$ Docente - Investigador, Grupo de Investigación en Ciencias Forenses y Salud, Tecnológico de Antioquia, Institución Universitaria. Medellín, Colombia. E-mail: man_alejo1781@yahoo.co.uk
} 


\section{INTRODUCCIÓN}

Uno de los aspectos más importantes de la entomología forense es el estudio de insectos y otros artrópodos asociados a cadáveres (CATTS \& GOFF, 1992; ANDERSON \& VANLAERHOVEN, 1996; BYRD \& CASTNER, 2001; AMENDT et al., 2004; NIHEI \& CARVALHO, 2007; OLIVEIRA \& VASCONCELOS, 2010). Estos buscan la materia orgánica en descomposición para alimentarse, reproducirse o depositar sus huevos o larvas. Es posible calcular el mínimo del intervalo post mortem (IPM) de un cuerpo en descomposición según el estado de desarrollo de los insectos que hayan sido encontrados en el lugar de los hechos (CATTS \& GOFF, 1992; BYRD \& CASTNER, 2001); es por esto que pueden aportar información para resolver casos médicolegales (AMENDT et al., 2004; YUSSEFF, 2006).

Una de las familias de moscas más frecuentes en la entomología forense, es Muscidae, especímenes inmaduros y adultos se encuentran comúnmente en las diferentes etapas de la descomposición cadavérica (GREENBERG, 1991; OLIVEIRA \& VASCONCELOS, 2010), y algunas de sus especies pueden ser utilizadas en el cálculo del IPM (CARVALHO et al., 2000; BYRD \& CASTNER, 2001; GRISALES et al., 2010; OLIVEIRA \& VASCONCELOS, 2010).

Muscidae es una familia que se encuentra en todas las regiones biogeográficas con cerca de 4000 mil especies (CARVALHO et al., 2005). Algunas de sus especies aparecen con frecuencia en trabajos de sucesión cadavérica y son reconocidas por su importancia forense, principalmente Biopyrellia bipuncta (Wiedemann, 1830), Musca domestica Linnaeus, 1758 y Synthesiomyia nudiseta (Wulp, 1883) en Argentina (HORENSTEIN et al., 2010), Brasil (CARVALHO \& MELLO-PATIU, 2008; BARBOSA et al., 2009; OLIVEIRA \& VASCONCELOS, 2010; VASCONCELOS \& ARAUJO, 2012) y en Colombia (BARRETO et al., 2002; SEGURA et al., 2009; GRISALES et al., 2010; RAMOS \& WOLFF, 2011).

La correcta identificación taxonómica de los especímenes, sin importar su estado de desarrollo, es esencial en cualquier investigación legal que incluya evidencia entomológica (HALL \& HUNTINGTON, 2009). Sin embargo, la mayoría de las claves para adultos en la actualidad solo utilizan características morfológicas externas (CARVALHO \& COURI, 2002; CARVALHO et al., 2002; CARVALHO \& MELLO-PATIU, 2008; NIHEI \& CARVALHO, 2009), esto requiere que los especímenes colectados se encuentren en buenas condiciones. Sin embargo, es común encontrarse con especímenes deteriorados por su colecta y preservación o solamente con partes de los especímenes adultos, lo que dificulta la identificación taxonómica.

Es conocido que las características de los genitales masculinos son una de las herramientas morfológicas más versátiles e importantes en la taxonomía de insectos (TUXEN, 1970; SONG \& BUCHELI, 2010). Los genitales masculinos poseen rasgos que son únicos a cada especie, especialmente entre especies relacionadas, y su utilidad en la identificación de especies ha sido ampliamente probada en diversos grupos (EBERHARD, 1985; HOSKEN \& STOCKLEY, 2004).

Este trabajo tiene como objetivo ilustrar y describir las estructuras genitales de los machos de las especies $B$. bipuncta, $M$. domestica y $S$. nudiseta, además de brindar una diagnosis de su morfología externa para complementar y facilitar el proceso de identificación y corroboración taxonómica de los especímenes. 


\section{MATERIALES Y MÉTODOS}

Se utilizaron 10 especímenes macho de las especies $B$. bipuncta, $M$. domestica y $S$. nudiseta pertenecientes a material depositado en la Colección Entomológica Tecnológico de Antioquia (CETdeA), Tecnológico de Antioquia - Institución Universitaria.

Para la descripción y diagnosis se siguió la terminología morfológica de McALPINE (1981), excepto: cerda calcar (CARVALHO, 1989) y las estructuras de los genitales según NIHEI \& CARVALHO (2007).

Para el análisis de los genitales, se retiró el abdomen de cada espécimen y se dispuso en $\mathrm{KOH}$ al $10 \%$ por un periodo de 48 horas para aclarar las estructuras, posteriormente se realizó neutralización con ácido acético y deshidratación con alcohol etanol a concentraciones de 30\%, 70\% y 96\%. Después, el abdomen se depositó en glicerina para realizar la disección y las ilustraciones. Se almacenaron los genitales en microviales con glicerina adheridos a su respectivo espécimen.

Las observaciones se realizaron por medio de estéreo microscopio Nikon SMZ 745T y microscopio Olympus CX31. Las ilustraciones se realizaron mediante cámara lúcida y luego se digitalizaron con el programa Adobe Illustrator ${ }^{\circledR}$.

\section{RESULTADOS}

\section{Biopyrellia bipuncta (Wiedemann, 1830)}

Diagnosis: Coloración azul oscuro metalizado, superficie con finas puntuaciones pilosas; alas con máculas sobre la vena humeral y sobre el ápice de Sc y $\mathrm{R}_{1}$. Macho con omatidios anterointernos agrandados, del mismo diámetro del ocelo anterior. Vibrisa reducida, insertada encima del margen oral. Dorsocentral 0+1. Poshumeral, parahumeral, postsutural intra-alar e intrapostalar ausente. Prosterno notablemente estrecho anteriormente y desnudo. Katepisternal 0+1. Merón y katepimeron desnudo. Espiráculo posterior desnudo sobre el margen posterior. Ala con la porción apical de la vena-raíz ventralmente setosa; nodo de Rs setosa dorsal y ventralmente; $\mathrm{R}_{4+5}$ desnuda dorsal y ventralmente; $\mathrm{M}$ dirigido hacia delante; $\mathrm{R}_{4+5}$. Esclerito subcostal desnudo. Calíptero inferior posteriormente alargado, extendiéndose bajo la base del escutelo. Tibia media con dos cerdas débiles posteroventrales, una supramedial y otra sobre el tercio apical. Calcar débil.

Genitales: Esternito V con pequeñas espinas por casi todo el margen posterior alcanzando la punta de cada lóbulo, cubierto con microtriquias sobre la mitad posterior, con cerdas largas dispersas (Figura 1). Placa cercal con ápice simple, redondeado, cubierto de cerdas delgadas casi 1/4 la longitud de la placa (Figura 2); ventralmente con lóbulo inferior casi encerrando el lóbulo por completo, con márgenes dentados hacia la parte distal (Figura 3). Surstilo como en la Figura 4, con pequeñas cerdas sobre el margen lateral y pequeñas espínulas sobre el margen distal. Hipandrio con margen anterior angosto, brazos laterales en su parte distal con proyección hacia adentro (Figura 5). Aedeago (Figura 6) con epifalo pequeño, triangular en vista lateral; parámero con cerdas dispersas en el ápice; distifalo campanulado en vista lateral, membrana latero-apical con pequeñas espinas hacia la 
parte media y distal; apodema del aedeago simple, alargado, redondeado en el ápice. Comentarios: Entre los especímenes examinados se encontraron variaciones en cuanto al tamaño y cantidad de espinas sobre el margen posterior del esternito V.

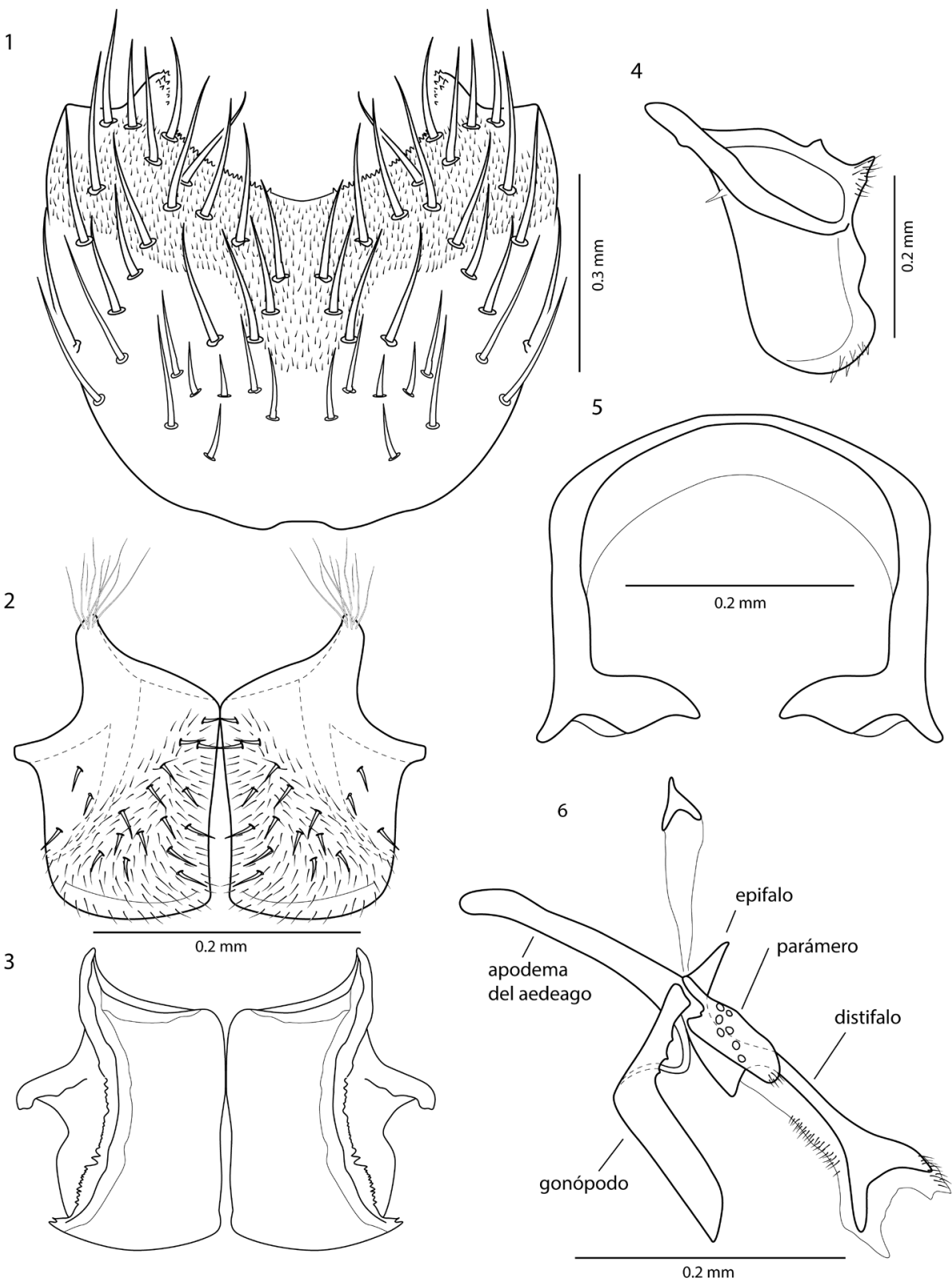

Figuras 1-6. B. bipuncta, genitales masculinos. 1: Esternito V, vista dorsal. 2: Placa cercal, vista dorsal. 3: Placa cercal, vista ventral. 4: Surstilo, vista lateral. 5: Hipandrio, vista dorsal. 6: Aedeago, vista lateral. 
Material examinado: 1 macho. COLOMBIA, Antioquia, Caldas, La Clara 0603'06,9”'N 75037'19,2'W. 9.II.2010. 1840 m. M.A. Ramírez, J. Durango, H. Areiza Cols. M02C2 (CETdeA 1933); 1 macho. COLOMBIA, Antioquia, Caldas, La Clara 0603'06,9'N 75³7'19,2'W. 10.III.2010. 1840 m. M.A. Ramírez, C. Rave Cols. M03C1 (CETdeA 2285); 4 machos. COLOMBIA, Antioquia, Copacabana, Ankón 06²2'07,1”N 75²9'22,3”W. 7.VII.2010. 1417 m. J. Durango, I. Cadavid, C. Rave Cols. 7CP1 (CETdeA 4032-4035); 3 machos. COLOMBIA, Antioquia, Medellín, Pajarito 06¹7'10,7'N 75³6'43,7'”W. 8.X.2010. 1929 m. M.A. Ramírez, I. Cadavid, C. Rave Cols. M10P2 (CETdeA 3331-3333); 1 macho. COLOMBIA, Antioquia, Medellín, Pajarito 06¹7'10,7’N 75³6’43,7'W. 10.XII.2010. 1929 m. I. Cadavid, A. Varela, C. Rave Cols. M12P2 (CETdeA 2771).

\section{Musca domestica Linnaeus, 1758}

Diagnosis: Coloración del tórax negro y pruinoso plateado. Macho holóptico o casi holóptico; omatidios anterointernos alargados. Arista alargada en el cuarto basal, plumosa. Acrosticales presuturales desarrolladas. Dorsocentral 2:4. Katepisternales 1:2. Anepimerón setoso. Prosterno setoso. Vena M fuertemente curvada hacia adelante apicalmente. Calíptero inferior ancho, subtruncado posteriormente, ángulo anteromedial extendiéndose un poco por debajo de la base del escutelo. Tibia media sin cerda ventral. Esternito 1 setoso.

Genitales: Esternito V con márgenes lisos, romboidal, puntas alargadas de cada lóbulo, en las esquinas posterolaterales con márgenes extendidos lateralmente, cerdas largas dispersas en los dos tercios posteriores (Figura 7). Placa cercal con ápice simple, cubierto con dispersas cerdas largas, microtriquias que cubren la placa con forma de W invertida (Figura 8); ventralmente con lóbulo ventral simple, hacia la mitad del lóbulo lateral con pequeña proyección de forma triangular dirigida hacia fuera (Figura 9). Surstilo como en la Figura 10, con pequeñas cerdas que cubren la proyección lateral, fila de cerdas largas sobre el margen lateral. Hipandrio con margen anterior amplio, ventralmente cóncavo, cubierto por pequeñas cerdas dispersas (Figura 11). Aedeago (Figura 12) con epifalo desarrollado, ápice redondeado en vista lateral, con membrana apical; parámero glabro; basifalo diagonalmente truncado en vista lateral, con membrana distal, sin cerdas; apodema del aedeago simple, curvado en vista lateral, ápice redondeado.

Comentarios: No se encontraron variaciones intraespecíficas en los especímenes examinados.

Material examinado: 4 machos. COLOMBIA, Antioquia, Medellín, Pajarito 06017'10,7”N 75036’43,7”'W. 12.III.2010. 1929 m. M.A. Ramírez Col. M03P2 (CETdeA 4036-4039); 3 machos. COLOMBIA, Antioquia, Copacabana, Ankón 06022'07,1”N 75029'22,3"W. 21.I.2011. 1417 m. I. Cadavid, A. Varela, C. Rave Cols. M13CP2 (CETdeA 2719-2721); 2 machos. COLOMBIA, Antioquia, Copacabana, Ankón 06²2'07,1'N 75²29'22,3'”W. 29.III.2011. 1417 m. J. Durango, H. Areiza, I. Cadavid Cols. M15CP1 (CETdeA); 1 macho. ANTIOQUIA, Copacabana, Ankón 06022'07,1”N 75²9'22,3”'W. 30.IX.2011. 1417 m. J. Durango, H. Areiza, I. Cadavid Cols. M21CP2 (CETdeA). 


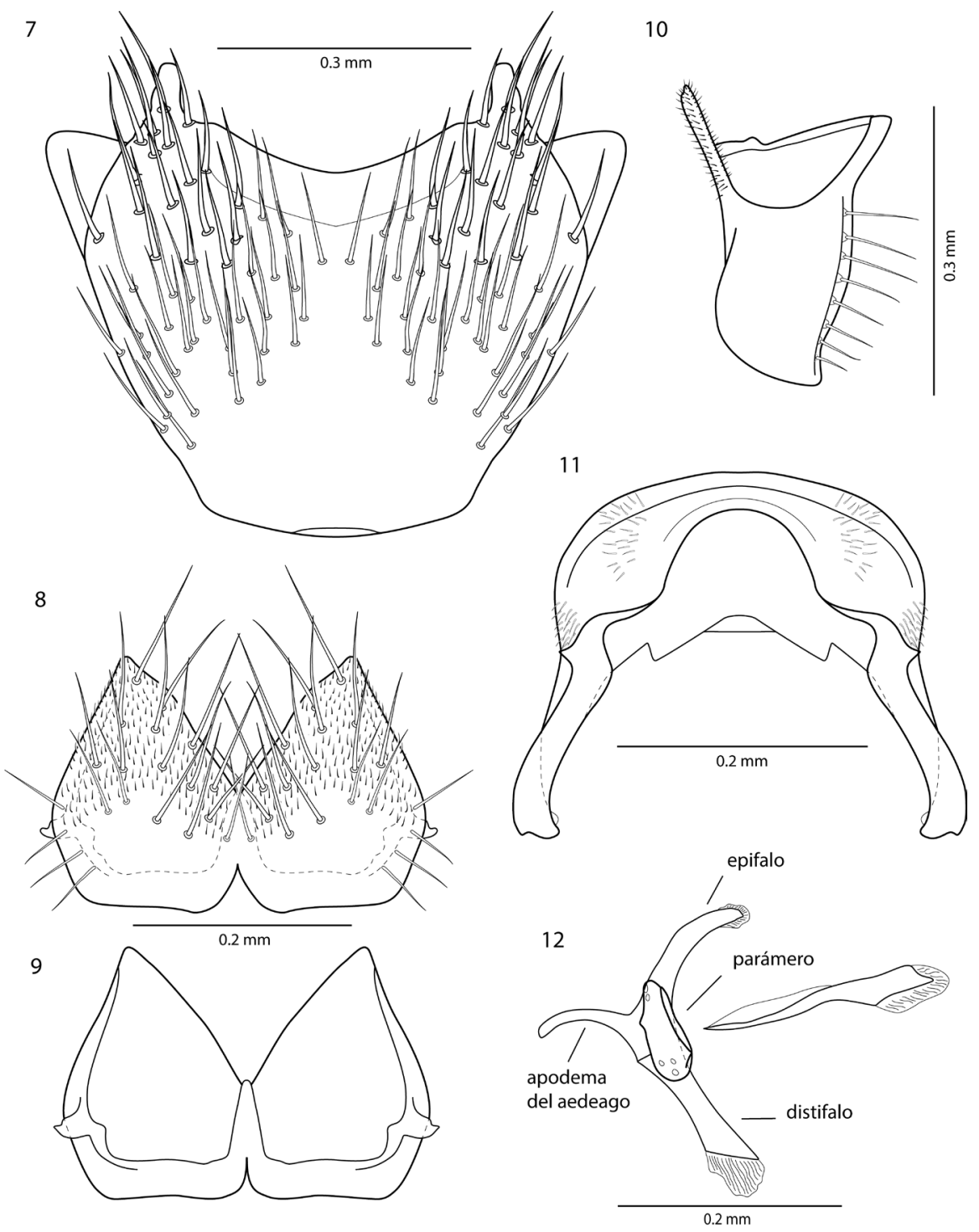

Figuras 7-12. M. domestica, genitales masculinos. 7: Esternito V, vista dorsal. 8: Placa cercal, vista dorsal. 9: Placa cercal, vista ventral. 10: Surstilo, vista lateral. 11: Hipandrio, vista dorsal. 12: Aedeago, vista lateral. 


\section{Synthesiomyia nudiseta (Wulp, 1883)}

Diagnosis: Macho holóptico; ojos desnudos. Arista pubescente, con cilios cortos. Borde facial sin sétulas. Acrostical presutural no diferenciada. Prealar fuerte. Katepimeron y anepimeron desnudos. Dorsocentrales 2:4. Katepisternales 1:2. Venas alares desnudas; M fuertemente curvada hacia adelante apicalmente. Calíptero inferior alargado; esternito 1 setoso.

Genitales: Esternito V con márgenes lisos, casi cuadrado, margen posterior ligeramente cóncavo hacia la mitad, margen anterior con pequeña protuberancia hacia la mitad de cada lóbulo (Figura 13). Placa cercal simple, cubierta por cerdas largas dispersas, ligeramente aguda sobre la punta de cada lóbulo, con proyecciones laterales (Figura 14); ventralmente con lóbulo inferior y lateral con forma rectangular, que cubren casi 1/3 de cada lóbulo, lóbulo interno terminando en puntas divergentes (Figura 15). Surstilo como en la Figura 16, pocas cerdas gruesas sobre la proyección interno-lateral (Figura 16). Hipandrio con margen anterior angosto, brazos laterales curvados hacia adentro (Figura 17). Aedeago (Figura 18) con epifalo grande, distalmente redondeado en vista lateral; parámero glabro, ligeramente curvado en su ápice; distifalo ligeramente campanulado en vista lateral, con espínulas sobre su ápice, sin membranas; apodema del aedeago simple, alargado, con ápice redondeado en vista lateral, sin membrana.

Comentarios: Entre los especímenes examinados se encontraron variaciones en la cantidad y longitud de las cerdas gruesas sobre la proyección interno-lateral del surstilo.

Material examinado: 1 macho. COLOMBIA, Antioquia, Medellín, Pajarito 06017'10,7’'N 75³6'43,7’'W. 8.X.2010. 1929 m. M.A. Ramírez, I. Cadavid, C. Rave Cols. M10P2 (CETdeA 3258); 5 machos. COLOMBIA, Antioquia, Copacabana, Ankón 06²2'07,1"N 75029'22,3”'W. 10.XII.2010. 1417 m. I. Cadavid, A. Varela, C. Rave Cols. M12CP2 (CETdeA 2666, 2669, 2704, 2711, 3111); 4 machos. COLOMBIA, Antioquia, Copacabana, Ankón 06022'07,1”N 75029'22,3”W. 31.VIII.2011. 1417 m. I. Cadavid, A. Varela Cols. M20CP1 (CETdeA).

\section{DISCUSIÓN}

Al realizar las revisiones de la bibliografía disponible sobre las tres especies examinadas, no se encontraron estudios descriptivos detallados de las estructuras genitales de especies tan comunes e históricamente estudiadas como lo es $M$. domestica. Con la descripción e ilustración de las características de los genitales de $B$. bipuncta, $M$. domestica y $S$. nudiseta, se muestra la utilidad de estructuras como el esternito $\mathrm{V}$, forma y quetotaxia de los surstilos, forma del hipandrio y del aedeago para su consideración como caracteres diagnósticos óptimos y que puedan ser incluidos en las claves de identificación taxonómicas. Sus características demuestran una adecuada diferenciación a nivel específico y evidencian un patrón constante independiente de cualquier tipo de variación de origen intrínseco o extrínseco que puedan afectar las estructuras morfológicas externas. 


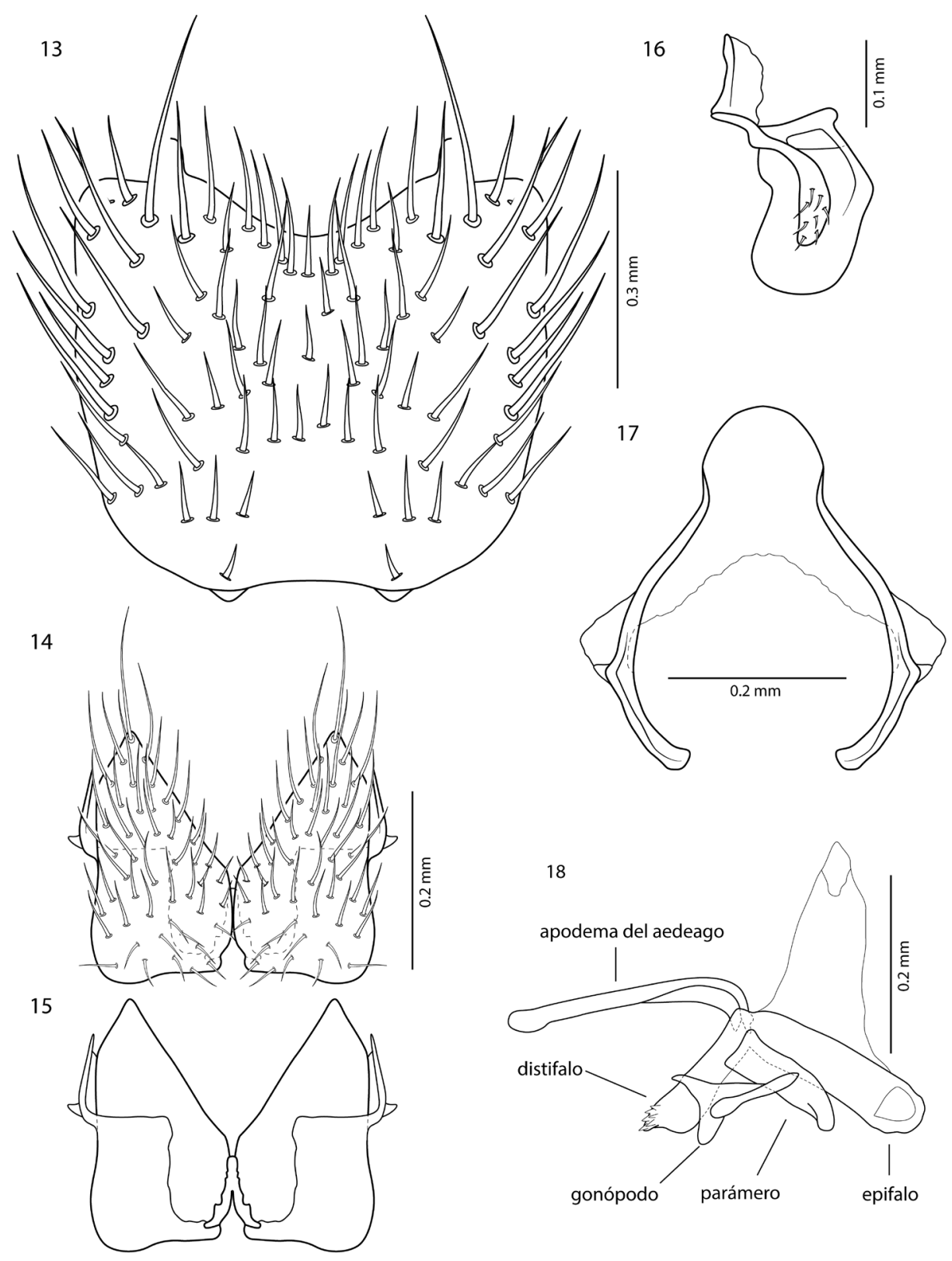

Figuras 13-18. S. nudiseta, genitales masculinos. 13: Esternito V, vista dorsal. 14: Placa cercal, vista dorsal. 15: Placa cercal, vista ventral. 16: Surstilo, vista lateral. 17: Hipandrio, vista dorsal. 18: Aedeago, vista lateral. 


\section{AGRADECIMIENTOS}

Agradecemos a John "Black" Quiroz y al Museo Entomológico Francisco Luís Gallego (MEFLG) por permitir el acceso a sus equipos y sus comentarios al documento. A Luz Miryam Gómez y Eduardo Amat por sus sugerencias al documento y al Grupo de Investigación en Ciencias Forenses y Salud, al semillero de INSECTA y al Comité de Investigación del Tecnológico de Antioquia -CODEI- por la financiación.

\section{BIBLIOGRAFÍA}

AMENDT, J., KRETTEK, R. \& ZEHNER, R., 2004.- Forensic entomology. Naturwissenschaften, 91 (2), 51-65. ANDERSON, G.S. \& VANLAERHOVEN, S., 1996.- Initial studies on insect succession on carrion in Southwestern British Columbia. J. Forensic Sci., 41 (4): 617-625.

BARBOSA, R.R., MELLO-PATIU, C.A., MELLO, R.P. \& QUEIROZ, M.M.C., 2009.- New records of calyptrate dipterans (Fanniidae, Muscidae and Sarcophagidae) associated with the decomposition of domestic pigs in Brazil. Mem. Inst. Oswaldo Cruz, 104 (6): 923-926.

BARRETO, M., BURBANO, M.E. \& BARRETO, P., 2002.- Flies (Calliphoridae, Muscidae) and beetles (Silphidae) from human cadavers in Cali, Colombia. Mem. Inst. Oswaldo Cruz, 97 (1): 137-138.

BYRD, J.H. \& CASTNER, J.L., 2001.- Forensic entomology: the utility of arthropods in legal investigations. CRC Press, Boca Raton.

CARVALHO, C.J., 1989.- Revisão das espécies e posição sistemática de Palpibracus Rondani (Diptera, Muscidae). Rev. Bras. Zool., 6 (2): 325-375.

CARVALHO, C.J. \& COURI, M.S., 2002.- Part I. Basal groups: 17-132 (in) CARVALHO, C.J. (ed.) Muscidae (Diptera) of the Neotropical Region: taxonomy. Editora Universidade Federal do Paraná, Curitiba.

CARVALHO, C.J., COURI, M.S, PONT, A.C., PAMPLONA, D. \& LOPES, S.M., 2005- A catalogue of the Muscidae (Diptera) of the Neotropical Region. Zootaxa, 860: 1-253.

CARVALHO, C.J. \& MELLO-PATIU, C.A., 2008.- Key to the adults of the most common forensic species of Diptera in South America. Rev. Bras. Entomol., 52 (3): 390-406.

CARVALHO, C.J., MOURA, M.O. \& RIBEIRO, P.B., 2002.- Chave para adultos de dípteros (Muscidae, Fanniidae, Anthomyiidae) associados ao ambiente humano no Brasil. Rev. Bras. Entomol., 46: 107-144.

CARVALHO, L.M., THYSSEN, P.J., LINHARES, A.X. \& PALHARES, F.A., 2000.- A checklist of arthropods associated with pig carrion and human corpses in southeastern brazil. Mem. Inst. Oswaldo Cruz, 95 (1): $135-138$

CATTS, E.P. \& GOFF, M.L., 1992.- Forensic entomology in criminal investigations. Annu. Rev. Entomol., 37: 253-272.

EBERHARD, W.G., 1985.- Sexual selection and animal genitalia. Harvard University Press, Cambridge, MA.

GREENBERG, B., 1991.- Flies as forensic indicators. J. Med. Entomology, 28: 565-577.

GRISALES, D., RUIZ, M. \& VILLEGAS, S., 2010.- Insects associated with exposed decomposing bodies in the Colombian Andean Coffee Region. Rev. Bras. Entomol., 54: 637-644.

HALL, R.D. \& HUNTINGTON, T.E., 2009.- Perceptions and Status of Forensic Entomology: 1-16 (in) BYRD, J.H. \& CASTNER, J.L. (eds.) Forensic Entomology: The Utility of Arthropods in Legal Investigations. Ed. CRC, Boca Raton, FL.

HORENSTEIN, M.B., LINHARES, A.X., FERRADAS, B.R. \& GARCÍA, D., 2010.- Decomposition and dipteran succession in pig carrion in central Argentina: ecological aspects and their importance in forensic science. Med. Vet. Entomol., 24 (1): 16-25.

HOSKEN, D.J. \& STOCKLEY, P., 2004.- Sexual selection and genital evolution. Trends. Ecol. Evol., 19: 87-93.

MCALPINE, J.F., 1981.- Morphology and terminology - adults: 9-63 (in) MACALPINE, J.F. (ed) Manual of Nearctic Diptera (Vol 1). Monograph No. 27. Ed. Research Branch Agriculture, Ottawa, Canada

NIHEI, S.S. \& CARVALHO, C.J., 2007.- Phylogeny and classification of Muscini (Diptera, Muscidae). Zool. J. Linn. Soc., 149 (4): 493-532.

2009.- The Muscini flies of the world (Diptera, Muscidae): Identification key and generic diagnoses. Zootaxa, 1976: 1-24

OLIVEIRA, T.C. \& VASCONCELOS, S.D., 2010.- Insects (Diptera) associated with cadavers at the Institute of Legal Medicine in Pernambuco, Brazil: implications for forensic entomology. Forensic Sci. Int., 198 (1-3): 97-102.

RAMOS, R. \& WOLFF, M., 2011.- Entomofauna cadavérica asociada a cerdos expuestos al sol y sombra, en el Piedemonte Amazónico Colombiano. Momentos de Ciencia, 8 (1): 45-53.

SEGURA, N.A., USAQUÉN, W., SÁNCHEZ, M.C., CHUAIRE, L. \& BELLO, F., 2009.- Succession pattern of cadaverous entomofauna in a semi-rural area of Bogotá, Colombia. Forensic Sci. Int., 187 (1): 66-72.

SONG, H. \& BUCHELI, S.R., 2010.- Comparison of phylogenetic signal between male genitalia and non-genital characters in insect systematics. Cladistics, 26: 23-35. 
TUXEN, S.L., 1970.- Taxonomist's glossary of genitalia in insects. Second edn. Scandinavian University Press, Copenhagen.

VASCONCELOS, S.D. \& ARAUJO, M.C.S., 2012.- Necrophagous species of Diptera and Coleoptera in northeastern Brazil: state of the art and challenges for the Forensic Entomologist. Rev. Bras. EntoYUSSEFF, S., 2006.- Entomología forense: los insectos en la escena del crimen. Revista Luna Azul, 23: 42-49. Recuperado de http://lunazul.ucaldas.edu.co/downloads/2f90012eRevista23_9.pdf 